ANNALES

POLONICI MATHEMATICI

$94.1(2008)$

\title{
The sharp version of a criterion for starlikeness related to the operator of Alexander
}

\author{
by RóBert SzÁsz (Corunca)
}

\begin{abstract}
The method of convolution is used to determine a sharp condition for starlikeness of analytic functions defined in the unit disc $U=\{z \in \mathbb{C}:|z|<1\}$ and having the property $f(0)=f^{\prime}(0)-1=0$. The integral version is given using the Alexander integral operator.
\end{abstract}

1. Introduction. Let $\mathcal{H}(U)$ be the class of analytic functions in the unit disc $U$ and let $\mathcal{A}=\left\{f \in \mathcal{H}(U): f(0)=f^{\prime}(0)-1=0\right\}$. The subclass of $\mathcal{A}$ consisting of starlike functions is denoted by $S^{*}$. It is well known that $S^{*}=\left\{f \in \mathcal{A}: \operatorname{Re}\left(z f^{\prime}(z) / f(z)\right)>0, z \in U\right\}$. In [2] it was proved that if $f \in \mathcal{A}$ satisfies the condition

$$
\operatorname{Re}\left\{f^{\prime}(z)+z f^{\prime \prime}(z)\right\}>-\frac{\pi^{2}-6}{24-\pi^{2}}=-0.273 \ldots, \quad z \in U,
$$

then $f \in S^{*}$. This result is equivalent to the result obtained in [1]. In [3] an improvement of the results obtained in [1] and [2] is given. In [1] the author conjectured that if $f \in \mathcal{A}$, then the greatest value of $c$ for which the inequality $\operatorname{Re}\left(f^{\prime}(z)+z f^{\prime \prime}(z)\right)>-c, z \in U$, implies $f \in S^{*}$, is

$$
c=\frac{2 \ln 2-1}{2(1-\ln 2)}=0.629 \ldots
$$

In this paper this conjecture will be confirmed.

2. Preliminaries. Let $A_{0}=\{f \in \mathcal{H}(U): f(0)=1\}$ and $\mathcal{P}=\left\{f \in A_{0}\right.$ : $\operatorname{Re}(f(z))>0$ for all $z \in U\}$. We will need the following definitions and lemmas to prove the main result. Let $f(z)=\sum_{k=0}^{\infty} a_{k} z^{k}$ and $g(z)=\sum_{k=0}^{\infty} b_{k} z^{k}$ be two analytic functions in $U$. The Hadamard product of $f$ and $g$ is defined

2000 Mathematics Subject Classification: Primary 30C45.

Key words and phrases: operator of Alexander, starlike functions, convolution.

This work was supported by the Research Foundation Sapientia. 
by

$$
(f * g)(z)=\sum_{k=0}^{\infty} a_{k} b_{k} z^{k}
$$

For $V \subset A_{0}$, the dual set of $V$ is

$$
V^{d}=\left\{g \in A_{0}:(f * g)(z) \neq 0 \text { for all } f \in V \text { and } z \in U\right\} .
$$

Let $h_{T} \in \mathcal{A}$ be the function defined by

$$
h_{T}(z)=\frac{i T \frac{z}{1-z}+\frac{z}{(1-z)^{2}}}{1+i T}, \quad T \in \mathbb{R} .
$$

The following two lemmas are established in [4]. if

Lemma 1 ([4, p. 94]). The function $f \in \mathcal{A}$ is in the class $S^{*}$ if and only

$$
\frac{f(z)}{z} * \frac{h_{T}(z)}{z} \neq 0 \quad \text { for all } z \in U \text { and } T \in \mathbb{R} .
$$

Lemma 2 ([4, p. 23]). The dual set of the class $\mathcal{P}=\left\{f \in A_{0}: \operatorname{Re}(f(z))\right.$ $>0, z \in U\}$ is

$$
\mathcal{P}^{d}=\left\{f \in A_{0}: \operatorname{Re}(f(z))>1 / 2, z \in U\right\} .
$$

Lemma 3. If the function $f:[0,1] \rightarrow(0, \infty)$ is increasing and continuous, then for every $a \in[0, \infty)$,

$$
\int_{0}^{1} \frac{x^{a+1}}{f(x)} d x \leq \frac{a+1}{a+2} \int_{0}^{1} \frac{x^{a}}{f(x)} d x .
$$

Proof.

$$
\int_{0}^{1} \frac{x^{a+1}}{f(x)} d x=\frac{a+1}{a+2} \int_{0}^{1} \frac{x^{a}}{f\left(x^{\frac{a+1}{a+2}}\right)} d x \leq \frac{a+1}{a+2} \int_{0}^{1} \frac{x^{a}}{f(x)} d x .
$$

Lemma 4. If $\theta \in(0,2 \pi)$ and $\beta>0$ then the following identity holds:

$$
\begin{aligned}
& \int_{0}^{\infty} \frac{x\left(e^{\theta x}+e^{(2 \pi-\theta) x}\right)}{\left(\beta^{2}+x^{2}\right)\left(e^{2 \pi x}-1\right)} d x+i \beta \int_{0}^{\infty} \frac{e^{(2 \pi-\theta) x}-e^{\theta x}}{\left(\beta^{2}+x^{2}\right)\left(e^{2 \pi x}-1\right)} d x \\
&=\frac{1}{2 \beta}+\sum_{k=1}^{\infty} \frac{e^{i \theta k}}{k+\beta} .
\end{aligned}
$$

Proof. Consider the function

$$
f(z)=\frac{e^{i \theta z}}{(\beta+z)\left(e^{2 \pi i z}-1\right)}, \quad \beta>0,
$$

where $\theta \in(0,2 \pi)$ is a fixed number. Let $\mathbb{N}$ denote the set of natural numbers. The contour $\Gamma(r, n)$ is constructed as follows: $\Gamma(r, n)=\gamma_{1} \cup \gamma_{3} \cup \gamma_{2} \cup \gamma_{4}$ where $\gamma_{1}(t)=R_{n} e^{i(\pi t-\pi / 2)}, \gamma_{2}(t)=r e^{i(-\pi t+\pi / 2)}, \gamma_{3}(t)=i R_{n}+t\left(i r-i R_{n}\right)$, 
$\gamma_{4}(t)=-i r+t\left(i r-i R_{n}\right), t \in[0,1], r \in(0,1)$ and $R_{n}=n+1 / 2, n \in \mathbb{N}$. The residue theorem implies that

$$
\int_{\Gamma(r, n)} f(z) d z=2 \pi i \sum_{k \in \mathbb{N}, 0<k<n+1 / 2} \operatorname{Res}(f, k) .
$$

A computation leads to

$$
\begin{aligned}
& \lim _{r \rightarrow 0} \int_{\gamma_{2}} f(z) d z=-i \pi \cdot \operatorname{Res}(f, 0), \\
& \operatorname{Res}\left(f, z_{k}\right)=\operatorname{Res}(f, k)=\frac{e^{i \theta k}}{2 \pi i(k+\beta)}, \quad k \in \mathbb{N} .
\end{aligned}
$$

We now give a detailed proof for the equality

$$
\lim _{R_{n} \rightarrow \infty} \int_{\gamma_{1}} f(z) d z=0 .
$$

Let $\left(\alpha_{n}\right)_{n \geq 2}$ be the sequence defined by

$$
\alpha_{n}=\frac{\ln n}{4 \pi^{2}(n+1 / 2)}, \quad n \geq 2 .
$$

We have

$$
\begin{aligned}
\left|\int_{\gamma_{1}} f(z) d z\right| \leq & \left|\int_{0}^{1 / 2-\alpha_{n}} f\left(\gamma_{1}(t)\right) \gamma_{1}^{\prime}(t) d t\right| \\
& +\left|\int_{1 / 2-\alpha_{n}}^{1 / 2+\alpha_{n}} f\left(\gamma_{1}(t)\right) \gamma_{1}^{\prime}(t) d t\right|+\left|\int_{1 / 2+\alpha_{n}}^{1} f\left(\gamma_{1}(t)\right) \gamma_{1}^{\prime}(t) d t\right|
\end{aligned}
$$

If $n>\beta$, the inequalities $\left|e^{2 \pi i \gamma_{1}(t)}-1\right| \geq e^{-2 \pi(n+1 / 2) \sin (\pi t-\pi / 2)}-1$ and $\left|\gamma_{1}(t)+\beta\right| \geq n+1 / 2-\beta$ for $t \in(0,1 / 2)$ imply that

$$
\begin{aligned}
\mid \int_{0}^{1 / 2-\alpha_{n}} f\left(\gamma_{1}(t)\right) & \gamma_{1}^{\prime}(t) d t \mid \\
& \leq \int_{0}^{1 / 2-\alpha_{n}} \frac{e^{-\theta(n+1 / 2) \sin (\pi t-\pi / 2)}}{e^{-2 \pi(n+1 / 2) \sin (\pi t-\pi / 2)}-1} \frac{n+1 / 2}{n+1 / 2-\beta} d t \\
& \leq \frac{1}{2} \frac{1}{e^{(2 \pi-\theta)(n+1 / 2) \sin \left(\pi \alpha_{n}\right)}-1} \frac{n+1 / 2}{n+1 / 2-\beta} \underset{n \rightarrow \infty}{\longrightarrow} 0 .
\end{aligned}
$$

Since $\lim _{t \rightarrow 1 / 2} e^{2 \pi i \gamma_{1}(t)}=-1$ and $\lim _{n \rightarrow \infty} \alpha_{n}=0$, there is a natural number $n_{0}$ such that $\left|e^{2 \pi i \gamma_{1}(t)}-1\right|>1$ for all $n>n_{0}$ and all $t \in\left[1 / 2-\alpha_{n}, 1 / 2+\alpha_{n}\right]$. 
Consequently, the second term on the right side of (5) can be estimated by

$$
\begin{aligned}
& \int_{1 / 2-\alpha_{n}}^{1 / 2+\alpha_{n}} \frac{n+1 / 2}{n+1 / 2-\beta} e^{-\theta(n+1 / 2) \sin (\pi t-\pi / 2)} d t \\
& \quad \leq 2 \alpha_{n} \frac{n+1 / 2}{n+1 / 2-\beta} e^{\theta(n+1 / 2) \sin \left(\pi \alpha_{n}\right)} \\
& \quad \leq 2 \alpha_{n} \frac{n+1 / 2}{n+1 / 2-\beta} e^{2 \pi(n+1 / 2) \pi \alpha_{n}}=2 \alpha_{n} \frac{n+1 / 2}{n+1 / 2-\beta} \sqrt{n} \underset{n \rightarrow \infty}{\longrightarrow} 0 .
\end{aligned}
$$

Finally, the inequalities

$\left|e^{i \theta \gamma_{1}(t)}\right| \leq e^{-\theta(n+1 / 2) \sin (\pi t-\pi / 2)}, \quad\left|e^{2 \pi i \gamma_{1}(t)}-1\right| \geq 1-e^{-2 \pi(n+1 / 2) \sin (\pi t-\pi / 2)}$

for $t \in\left[1 / 2+\alpha_{n}, 1\right]$ imply that the third term on the right side on (5) is at most

$$
\begin{aligned}
& \int_{1 / 2+\alpha_{n}}^{1} \frac{e^{-\theta(n+1 / 2) \sin (\pi t-\pi / 2)}}{1-e^{-2 \pi(n+1 / 2) \sin (\pi t-\pi / 2)}} \frac{n+1 / 2}{n+1 / 2-\beta} d t \\
& \leq \frac{1}{2} \frac{e^{-\theta(n+1 / 2) \sin \left(\pi \alpha_{n}\right)}}{1-e^{-2 \pi(n+1 / 2) \sin \left(\pi \alpha_{n}\right)}} \frac{n+1 / 2}{n+1 / 2-\beta} \underset{n \rightarrow \infty}{\longrightarrow} 0 .
\end{aligned}
$$

Summarizing, we see that (4) follows from (5)-(8). From (2)-(4) we obtain

$$
\lim _{\substack{R_{n} \rightarrow \infty \\ r \rightarrow 0}}\left(\int_{\gamma_{3}} f(z) d z+\int_{\gamma_{4}} f(z) d z\right)=\frac{1}{2 \beta}+\sum_{k=1}^{\infty} \frac{e^{i \theta k}}{k+\beta},
$$

which is equivalent to

$$
\begin{aligned}
\int_{0}^{\infty} \frac{x\left(e^{\theta x}+e^{(2 \pi-\theta) x}\right)}{\left(\beta^{2}+x^{2}\right)\left(e^{2 \pi x}-1\right)} d x+i \beta \int_{0}^{\infty} \frac{e^{(2 \pi-\theta) x}-e^{\theta x}}{\left(\beta^{2}+x^{2}\right)\left(e^{2 \pi x}-1\right)} d x \\
=\frac{1}{2 \beta}+\sum_{k=1}^{\infty} \frac{e^{i \theta k}}{k+\beta} .
\end{aligned}
$$

Lemma 5. Let $f:[a, b] \times[0, \infty) \rightarrow \mathbb{R}$ be a continuous function and let $g:[0, \infty) \rightarrow \mathbb{R}$ be an increasing non-constant function. Suppose that:

(i) $\left|f\left(\beta_{1}, x\right)-f\left(\beta_{2}, x\right)\right| \leq\left|\beta_{1}-\beta_{2}\right| q(x)$ for all $\beta_{1}, \beta_{2} \in[a, b]$ and $x \in$ $[0, \infty)$, where $q:[0, \infty) \rightarrow[0, \infty)$ is a continuous function and the improper integral $\int_{0}^{\infty} q(x) d g(x)$ is convergent,

(ii) the function $f$ is differentiable with respect to the first variable, and $\partial f / \partial \beta:(a, b) \times[0, \infty) \rightarrow \mathbb{R}$ is continuous,

(iii) the parametric improper integral $\int_{0}^{\infty} \frac{\partial f}{\partial \beta}(\beta, x) d g(x)$ is uniformly convergent on every compact subset of $(a, b)$. 
Then the function

$$
h:[a, b] \rightarrow \mathbb{R}, \quad h(\beta)=\int_{0}^{\infty} f(\beta, x) d g(x),
$$

is differentiable at every $\beta_{0} \in(a, b)$ and

$$
h^{\prime}\left(\beta_{0}\right)=\int_{0}^{\infty} \frac{\partial f}{\partial \beta}\left(\beta_{0}, x\right) d g(x) .
$$

Proof. Let $\beta_{0} \in(a, b)$. There is a $\delta>0$ for which $I=\left[\beta_{0}-\delta, \beta_{0}+\delta\right] \subset$ $(a, b)$.

According to (i) and (iii) there exists an $N>0$ so that $g(N)>g(0)$ and

$$
\begin{array}{r}
\left|\int_{M}^{\infty} \frac{\partial f}{\partial \beta}(\beta, x) d g(x)\right|<\frac{\varepsilon}{3} \text { and } \quad\left|\int_{M}^{\infty} q(x) d g(x)\right|<\frac{\varepsilon}{3} \\
\quad \text { for } M \in[N, \infty) \text { and } \beta \in I .
\end{array}
$$

Condition (i) also implies that

$$
\int_{N}^{\infty}\left|\frac{f(\beta, x)-f\left(\beta_{0}, x\right)}{\beta-\beta_{0}}\right| d g(x) \leq \int_{N}^{\infty} q(x) d g(x) .
$$

The (Lagrange) mean value theorem implies that there is a $\theta_{(\beta, x)} \in(0,1)$ such that

$$
\frac{f(\beta, x)-f\left(\beta_{0}, x\right)}{\beta-\beta_{0}}=\frac{\partial f}{\partial \beta}\left(\beta+\theta_{(\beta, x)}\left(\beta_{0}-\beta\right), x\right) .
$$

Since $\partial f / \partial \beta$ is uniformly continuous on the compact set $I \times[0, N]$, there is a $\delta^{\prime} \in(0, \delta)$ such that if $\left|\beta-\beta_{0}\right|<\delta^{\prime}$, then

$$
\left|\frac{\partial f}{\partial \beta}(\beta, x)-\frac{\partial f}{\partial \beta}\left(\beta_{0}, x\right)\right|<\frac{\varepsilon}{3(g(N)-g(0))} \quad \text { for } x \in[0, N] .
$$

If $\left|\beta-\beta_{0}\right|<\delta^{\prime}$, then $\left|\beta+\theta_{(\beta, x)}\left(\beta_{0}-\beta\right)-\beta_{0}\right|<\delta^{\prime}$, and so

$$
\left|\frac{f(\beta, x)-f\left(\beta_{0}, x\right)}{\beta-\beta_{0}}-\frac{\partial f}{\partial \beta}\left(\beta_{0}, x\right)\right|<\frac{\varepsilon}{3(g(N)-g(0))} \quad \text { for } x \in[0, N] .
$$

Provided that $0<\left|\beta-\beta_{0}\right|<\delta^{\prime}$, inequalities (10)-(12) imply

$$
\begin{aligned}
\mid \frac{h(\beta)-h\left(\beta_{0}\right)}{\beta-\beta_{0}}-\int_{0}^{\infty} \frac{\partial f}{\partial \beta} & \left(\beta_{0}, x\right) d g(x) \mid \\
= & \left|\int_{0}^{\infty}\left(\frac{f(\beta, x)-f\left(\beta_{0}, x\right)}{\beta-\beta_{0}}-\frac{\partial f}{\partial \beta}\left(\beta_{0}, x\right)\right) d g(x)\right|
\end{aligned}
$$




$$
\begin{aligned}
& \leq \int_{0}^{N}\left|\frac{f(\beta, x)-f\left(\beta_{0}, x\right)}{\beta-\beta_{0}}-\frac{\partial f}{\partial \beta}\left(\beta_{0}, x\right)\right| d g(x) \\
&+\int_{N}^{\infty}\left|\frac{f(\beta, x)-f\left(\beta_{0}, x\right)}{\beta-\beta_{0}}\right| d g(x)+\left|\int_{N}^{\infty} \frac{\partial f}{\partial \beta}\left(\beta_{0}, x\right) d g(x)\right| \\
& \leq \varepsilon / 3+\varepsilon / 3+\varepsilon / 3=\varepsilon,
\end{aligned}
$$

and the proof is complete.

REMARK 1. If we put, in Lemma $5, g(x)=[x]$ where $[x]$ denotes the integer part of $x$, then the improper integral $\int_{0}^{\infty} f(\beta, x) d g(x)$ and the series $\sum_{n=1}^{\infty} f(\beta, n)$ are convergent or divergent at the same time. If they are convergent then

$$
\int_{0}^{\infty} f(\beta, x) d g(x)=\sum_{n=1}^{\infty} f(\beta, n) .
$$

Hence we obtain an analogous lemma for the differentiability of series.

\section{The main result}

ThEOREM 1. If $f \in \mathcal{A}$ and

$$
\operatorname{Re}\left(f^{\prime}(z)+z f^{\prime \prime}(z)\right)>\frac{1-2 \ln 2}{2(1-\ln 2)} \quad \text { for } z \in U,
$$

then $f$ belongs to the class $S^{*}$, and the result is sharp.

Proof. The condition $\operatorname{Re}\left(f^{\prime}(z)+z f^{\prime \prime}(z)\right)>-\alpha, z \in U$, is equivalent to

$$
\operatorname{Re}\left(\frac{\alpha+f^{\prime}(z)+z f^{\prime \prime}(z)}{1+\alpha}\right)>0, \quad z \in U,
$$

which can be rewritten as

$$
\frac{\alpha+f^{\prime}+z f^{\prime \prime}}{1+\alpha} \in \mathcal{P} .
$$

According to the representation theorem of Herglotz there is a probability measure $\mu$ on $[0,2 \pi]$ so that

$$
\frac{\alpha+f^{\prime}(z)+z f^{\prime \prime}(z)}{1+\alpha}=\int_{0}^{2 \pi} \frac{1+e^{-i t} z}{1-e^{-i t} z} d \mu(t)
$$

hence, if $f(z)=z+\sum_{n=1}^{\infty} a_{n} z^{n}$, then

$$
1+\frac{1}{1+\alpha} \sum_{n=2}^{\infty} n^{2} a_{n} z^{n-1}=1+2 \sum_{n=1}^{\infty} z^{n} \int_{0}^{2 \pi} e^{-i n t} d \mu(t) .
$$


This implies $a_{n}=\frac{2(1+\alpha)}{n^{2}} \int_{0}^{2 \pi} e^{-i(n-1) t} d \mu(t)$ for $n \in \mathbb{N}, n \geq 2$ and

$$
f(z)=z+2(1+\alpha) \sum_{n=2}^{\infty} \frac{z^{n}}{n^{2}} \int_{0}^{2 \pi} e^{-i(n-1) t} d \mu(t) .
$$

By Lemma 1, we have to prove that

$$
\begin{aligned}
\frac{f(z)}{z} * & \frac{h_{T}(z)}{z}=\left(1+2 \sum_{n=1}^{\infty} z^{n} \int_{0}^{2 \pi} e^{-i n t} d \mu(t)\right) \\
& *\left(1+(1+\alpha) \sum_{n=1}^{\infty} \frac{1+n+i T}{(1+i T)(n+1)^{2}} z^{n}\right) \neq 0, \quad z \in U, T \in \mathbb{R} .
\end{aligned}
$$

The function defined by the development $1+2 \sum_{n=1}^{\infty} z^{n} \int_{0}^{2 \pi} e^{-i n t} d \mu(t)$ belongs to the class $\mathcal{P}$ for every $z \in U$ and every probability measure $\mu$. From Lemma 2 and (13) it follows that $f$ is starlike if and only if the function

$$
g(z)=1+(1+\alpha) \sum_{n=1}^{\infty} \frac{1+n+i T}{(1+i T)(n+1)^{2}} z^{n}, \quad z \in U,
$$

belongs to the dual set $\mathcal{P}^{d}$, that is,

$$
\operatorname{Re}\left(1+(1+\alpha) \sum_{n=1}^{\infty} \frac{1+n+i T}{(1+i T)(n+1)^{2}} z^{n}\right)>\frac{1}{2}, \quad z \in U, T \in \mathbb{R} .
$$

This inequality is equivalent to

$$
\operatorname{Re}\left(\frac{1}{2(1+\alpha)}+\sum_{n=1}^{\infty} \frac{1+n+i T}{(1+i T)(n+1)^{2}} z^{n}\right)>0, \quad z \in U, T \in \mathbb{R} .
$$

We will determine

$$
\inf _{\substack{\theta \in[0,2 \pi] \\ T \in \mathbb{R}}} \operatorname{Re}\left(\sum_{n=1}^{\infty} \frac{1+n+i T}{(1+i T)(n+1)^{2}} e^{i n \theta}\right)=: m .
$$

If $1 /(2(1+\alpha))=-m$, then $\alpha$ will be the greatest real number for which (14) is valid. Let us introduce the notation

$$
\begin{aligned}
M(\theta, T)= & \operatorname{Re}\left(\sum_{n=1}^{\infty} \frac{1+n+i T}{(1+i T)(n+1)^{2}} e^{i n \theta}\right) \\
= & \operatorname{Re}\left(-1+\frac{1}{1+T^{2}} \sum_{n=0}^{\infty} \frac{e^{i n \theta}}{n+1}+\frac{T^{2}}{1+T^{2}} \sum_{n=0}^{\infty} \frac{e^{i n \theta}}{(n+1)^{2}}\right. \\
& \left.-\frac{i T}{1+T^{2}} \sum_{n=0}^{\infty} \frac{e^{i n \theta}}{n+1}+\frac{i T}{1+T^{2}} \sum_{n=0}^{\infty} \frac{e^{i n \theta}}{(n+1)^{2}}\right)
\end{aligned}
$$


The following identities hold for every $\theta \in(0,2 \pi)$ :

$$
\begin{aligned}
\sum_{n=0}^{\infty} \frac{e^{i n \theta}}{n+1}= & \int_{0}^{1} \frac{1-t \cos \theta}{1+t^{2}-2 t \cos \theta} d t+i \sin \theta \int_{0}^{1} \frac{t}{1+t^{2}-2 t \cos \theta} d t \\
\sum_{n=0}^{\infty} \frac{e^{i n \theta}}{(n+1)^{2}}= & \int_{0}^{1} \int_{0}^{1} \frac{1-t x \cos \theta}{1+t^{2} x^{2}-2 t x \cos \theta} d t d x \\
& +i \sin \theta \int_{0}^{1} \int_{0}^{1} \frac{t x}{1+t^{2} x^{2}-2 t x \cos \theta} d t d x,
\end{aligned}
$$

and imply

$$
\begin{aligned}
M(\theta, T)= & -1+\frac{1}{1+T^{2}} \int_{0}^{1} \frac{1-t \cos \theta}{1+t^{2}-2 t \cos \theta} d t \\
& +\frac{T^{2}}{1+T^{2}} \int_{0}^{1} \int_{0}^{1} \frac{1-t x \cos \theta}{1+t^{2} x^{2}-2 t x \cos \theta} d t d x \\
& +\frac{T \sin \theta}{1+T^{2}} \int_{0}^{1} \frac{t}{1+t^{2}-2 t \cos \theta} d t \\
& -\frac{T \sin \theta}{1+T^{2}} \int_{0}^{1} \int_{0}^{1} \frac{t x}{1+t^{2} x^{2}-2 t x \cos \theta} d t d x .
\end{aligned}
$$

Using the identities

$\int_{0}^{1} \frac{1-t \cos \theta}{1+t^{2}-2 \cos \theta} d t=\int_{0}^{1} \frac{1}{1+t} d t+(1+\cos \theta) \int_{0}^{1} \frac{t(1-t)}{(1+t)\left(1+t^{2}-2 t \cos \theta\right)} d t$,

$\int_{0}^{1} \int_{0}^{1} \frac{1-t x \cos \theta}{1+t^{2} x^{2}-2 t x \cos \theta} d t d x=\int_{0}^{1} \int_{0}^{1} \frac{1}{1+t x} d t d x$

$$
+(1+\cos \theta) \int_{0}^{1} \int_{0}^{1} \frac{t x(1-t x)}{(1+t x)\left(1+t^{2} x^{2}-2 t x \cos \theta\right)} d t d x, \quad \theta \in(0,2 \pi),
$$

it follows that

$$
M(\theta, T)=-1+\int_{0}^{1} \frac{1}{1+t} d t+\frac{1}{1+T^{2}} L_{1}(\theta, T)
$$

where

$$
\begin{aligned}
L_{1}(\theta, T):= & (1+\cos \theta) \int_{0}^{1} \frac{t(1-t)}{(1+t)\left(1+t^{2}-2 t \cos \theta\right)} d t \\
& +T \sin \theta \int_{0}^{1} \int_{0}^{1} \frac{t(1-x)\left(1-t^{2} x\right)}{\left(1+t^{2}-2 t \cos \theta\right)\left(1+t^{2} x^{2}-2 t x \cos \theta\right)} d t d x
\end{aligned}
$$




$$
\begin{aligned}
& +T^{2}\left(\int_{0}^{1} \int_{0}^{1} \frac{t(1-x)}{(1+t)(1+t x)} d t d x\right. \\
& \left.+(1+\cos \theta) \int_{0}^{1} \int_{0}^{1} \frac{t x(1-t x)}{(1+t x)\left(1+t^{2} x^{2}-2 t x \cos \theta\right)} d t d x\right) .
\end{aligned}
$$

If we can prove that

$$
\inf _{\substack{\theta \in[0,2 \pi] \\ T \in \mathbb{R}}} L_{1}(\theta, T)=0
$$

then we will obtain

$$
\inf _{\substack{\theta \in[0,2 \pi] \\ T \in \mathbb{R}}} M(\theta, T)=-1+\ln 2 .
$$

Since $L_{1}(\theta, T)$ is a polynomial of the second degree in $T$ with a positive dominant coefficient and $L_{1}(\pi, 0)=0$, it is sufficient to show that the discriminant of $L_{1}(\theta, T)$ satisfies $\Delta_{L_{1}}(\theta) \leq 0$ for all $\theta \in(0,2 \pi)$. Now,

$$
\begin{aligned}
\Delta_{L_{1}}(\theta) & =\sin ^{2} \theta\left(\int_{0}^{1} \int_{0}^{1} \frac{t(1-x)\left(1-t^{2} x\right)}{\left(1+t^{2}-2 t \cos \theta\right)\left(1+t^{2} x^{2}-2 t x \cos \theta\right)} d t d x\right)^{2} \\
& -4(1+\cos \theta) \int_{0}^{1} \frac{t(1-t)}{(1+t)\left(1+t^{2}-2 t \cos \theta\right)} d t\left(\int_{0}^{1} \frac{t(1-x)}{(1+t)(1+t x)} d t d x\right. \\
& \left.+(1+\cos \theta) \int_{0}^{1} \int_{0}^{1} \frac{t x(1-t x)}{(1+t x)\left(1+t^{2} x^{2}-2 t x \cos \theta\right)} d t d x\right) .
\end{aligned}
$$

Because $\sup _{\theta \in[0,2 \pi]} \Delta_{L_{1}}(\theta)=\sup _{\theta \in[0, \pi]} \Delta_{L_{1}}(\theta)$ we can restrict to $\theta \in[0, \pi]$. Some calculations lead to

$$
\begin{aligned}
\Delta_{L_{1}}(\theta)= & 4 \cos ^{2} \frac{\theta}{2}\left[\sin ^{2} \frac{\theta}{2}\left(\int_{0}^{1} \int_{0}^{1} \frac{t(1-x)\left(1-t^{2} x\right)}{\left(1+t^{2}-2 t \cos \theta\right)\left(1+t^{2} x^{2}-2 t x \cos \theta\right)} d t d x\right)^{2}\right. \\
& -2 \int_{0}^{1} \frac{t(1-t)}{(1+t)\left(1+t^{2}-2 t \cos \theta\right)} d t\left(\int_{0}^{1} \int_{0}^{1} \frac{t(1-x)}{(1+t)(1+t x)} d t d x\right. \\
& \left.\left.+(1+\cos \theta) \int_{0}^{1} \int_{0}^{1} \frac{t x(1-t x)}{(1+t x)\left(1+t^{2} x^{2}-2 t x \cos \theta\right)} d t d x\right)\right]
\end{aligned}
$$

We will prove the inequality

$$
\begin{aligned}
\sin ^{2} \frac{\theta}{2}\left(\int_{0}^{1} \int_{0}^{1} \frac{t(1-x)\left(1-t^{2} x\right)}{\left(1+t^{2}-2 t \cos \theta\right)\left(1+t^{2} x^{2}-2 t x \cos \theta\right)} d t d x\right)^{2} \\
\quad \leq 2 \int_{0}^{1} \frac{t(1-t)}{(1+t)\left(1+t^{2}-2 t \cos \theta\right)} d t \int_{0}^{1} \frac{t(1-x)}{(1+t)(1+t x)} d t d x
\end{aligned}
$$


for $\theta \in[\pi / 2, \pi]$, which implies the claimed condition $\Delta_{L_{1}}(\theta) \leq 0$ in the case $\theta \in[\pi / 2, \pi]$.

If $\theta \in[\pi / 2, \pi]$ the function $g:[0,1] \rightarrow \mathbb{R}, g(t)=(1+t)\left(1+t^{2}-2 t \cos \theta\right)$, is strictly increasing and so Lemma 3 implies that

$$
\frac{2}{3} \int_{0}^{1} \frac{t}{(1+t)\left(1+t^{2}-2 t \cos \theta\right)} d t \geq \int_{0}^{1} \frac{t^{2}}{(1+t)\left(1+t^{2}-2 t \cos \theta\right)} d t,
$$

which is equivalent to

$$
\begin{aligned}
\int_{0}^{1} \frac{t-t^{2}}{(1+t)\left(1+t^{2}-2 t \cos \theta\right)} d t \\
\geq \frac{2}{3} \int_{0}^{1} \int_{0}^{1} \frac{t(1-x)}{(1+t)\left(1+t^{2}-2 t \cos \theta\right)} d t d x, \quad \theta \in[\pi / 2, \pi] .
\end{aligned}
$$

This shows that it is enough to prove

$$
\begin{aligned}
\sin ^{2} \frac{\theta}{2} & \left(\int_{0}^{1} \int_{0}^{1} \frac{t(1-x)\left(1-t^{2} x\right)}{\left(1+t^{2}-2 t \cos \theta\right)\left(1+t^{2} x^{2}-2 t x \cos \theta\right)} d t d x\right)^{2} \\
& \leq \frac{4}{3} \int_{0}^{1} \int_{0}^{1} \frac{t(1-x)}{(1+t)\left(1+t^{2}-2 t \cos \theta\right)} d t d x \int_{0}^{1} \int_{0}^{1} \frac{t(1-x)}{(1+t)(1+t x)} d t d x .
\end{aligned}
$$

This will yield (15) in the case $\theta \in[\pi / 2, \pi]$.

The inequality of Cauchy-Schwarz implies

$$
\begin{aligned}
& \left(\int_{0}^{1} \int_{0}^{1} \frac{t(1-x)}{(1+t) \sqrt{\left(1+t^{2}-2 t \cos \theta\right)(1+t x)}} d t d x\right)^{2} \\
& \quad \leq \int_{0}^{1} \int_{0}^{1} \frac{t(1-x)}{(1+t)\left(1+t^{2}-2 t \cos \theta\right)} d t d x \int_{0}^{1} \int_{0}^{1} \frac{t(1-x)}{(1+t)(1+t x)} d t d x
\end{aligned}
$$

for $\theta \in[0, \pi]$. If we prove

$$
\begin{aligned}
& \left|\sin \frac{\theta}{2}\right| \int_{0}^{1} \int_{0}^{1} \frac{t(1-x)\left(1-t^{2} x\right)}{\left(1+t^{2}-2 t \cos \theta\right)\left(1+t^{2} x^{2}-2 t x \cos \theta\right)} d t d x \\
& \quad \leq \frac{2}{\sqrt{3}} \int_{0}^{1} \int_{0}^{1} \frac{t(1-x)}{(1+t) \sqrt{\left(1+t^{2}-2 t \cos \theta\right)(1+t x)}} d t d x, \quad \theta \in[\pi / 2, \pi],
\end{aligned}
$$

then this inequality and (18) imply (17). 
Inequality (19) can be deduced from

$$
\begin{aligned}
& \frac{t(1-x)\left(1-t^{2} x\right) \sin \frac{\theta}{2}}{\left(1+t^{2}-2 t \cos \theta\right)\left(1+t^{2} x^{2}-2 t x \cos \theta\right)} \\
& \leq \frac{2}{\sqrt{3}} \frac{t(1-x)}{(1+t) \sqrt{\left(1+t^{2}-2 t \cos \theta\right)(1+t x)}}
\end{aligned}
$$

for $\theta \in[\pi / 2, \pi]$ and $t, x \in[0,1]$. The latter inequality is valid because through direct calculations it can be shown that

$$
\frac{\sin \frac{\theta}{2}}{\sqrt{1+t^{2}-2 t \cos \theta}} \leq \frac{1}{1+t} \text { and } \frac{1-t^{2} x}{1+t^{2} x^{2}-2 t x \cos \theta} \leq \frac{2}{\sqrt{3(1+t x)}}
$$

for $\theta \in[\pi / 2, \pi, t \in[0,1]$.

In order to finish the proof, we must also prove that $L_{1}(\theta, T) \geq 0$ for $\theta \in[0, \pi / 2]$ and $T \in \mathbb{R}$. To do this, we will apply Lemmas 4 and 5 .

We have proved in Lemma 4 the identity

$$
\begin{aligned}
\int_{0}^{\infty} \frac{x\left(e^{\theta x}+e^{(2 \pi-\theta) x}\right)}{\left(\beta^{2}+x^{2}\right)\left(e^{2 \pi x}-1\right)} d x+i \beta \int_{0}^{\infty} \frac{e^{(2 \pi-\theta) x}-e^{\theta x}}{\left(\beta^{2}+x^{2}\right)\left(e^{2 \pi x}-1\right)} d x & =\frac{1}{2 \beta}+\sum_{k=1}^{\infty} \frac{e^{i \theta k}}{k+\beta} .
\end{aligned}
$$

Let $f_{1}, f_{2}$ be the functions defined by

$$
\begin{aligned}
f_{1}, f_{2}:[1 / 2,3 / 2] \times[0, \infty) \rightarrow \mathbb{R}, \quad f_{1}(\beta, x) & =\frac{x\left(e^{\theta x}+e^{(2 \pi-\theta) x}\right)}{\left(\beta^{2}+x^{2}\right)\left(e^{2 \pi x}-1\right)}, \\
f_{2}(\beta, x) & =\frac{e^{(2 \pi-\theta) x}-e^{\theta x}}{\left(\beta^{2}+x^{2}\right)\left(e^{2 \pi x}-1\right)} .
\end{aligned}
$$

Since

$$
\begin{array}{r}
\left|f_{k}\left(\beta_{1}, x\right)-f_{k}\left(\beta_{2}, x\right)\right| \leq\left|\beta_{1}-\beta_{2}\right| \frac{3 x\left(e^{2 \pi x}+1\right)}{\left((1 / 2)^{2}+x^{2}\right)^{2}\left(e^{2 \pi x}-1\right)}=:\left|\beta_{1}-\beta_{2}\right| q(x), \\
x \in(0, \infty), \beta \in[1 / 2,3 / 2], k=1,2,
\end{array}
$$

and the integral $\int_{0}^{\infty} q(x) d x$ is convergent, the functions $f_{1}, f_{2}$ satisfy condition (i) of Lemma 5. Condition (ii) is automatically satisfied. Since

$$
\begin{aligned}
& \left|\frac{\partial f_{k}}{\partial \beta}(\beta, x)\right| \leq \frac{3 x\left(e^{2 \pi x}+1\right)}{\left((1 / 2)^{2}+x^{2}\right)^{2}\left(e^{2 \pi x}-1\right)}, \\
& \quad x \in(0, \infty), \beta \in[1 / 2,3 / 2], k=1,2,
\end{aligned}
$$

according to a theorem due to Weierstrass the convergence of the integral

$$
\int_{0}^{\infty} \frac{x\left(e^{2 \pi x}+1\right)}{\left((1 / 2)^{2}+x^{2}\right)^{2}\left(e^{2 \pi x}-1\right)} d x
$$


implies that the integral $\int_{0}^{\infty} \frac{\partial f}{\partial \beta}(\beta, x) d x$ is uniformly convergent with respect to $\beta$ on $[1 / 2,3 / 2]$, and so condition (iii) of Lemma 5 is also satisfied.

If $\beta \in(1 / 2,3 / 2)$ then from Lemma 5 and Remark 1 we see that both sides of the equality (20) can be differentiated with respect to $\beta$ :

$$
\begin{aligned}
& 2 \beta \int_{0}^{\infty} \frac{x\left(e^{\theta x}+e^{(2 \pi-\theta) x}\right)}{\left(\beta^{2}+x^{2}\right)^{2}\left(e^{2 \pi x}-1\right)} d x \\
& \quad+i \int_{0}^{\infty} \frac{\left(\beta^{2}-x^{2}\right)\left(e^{(2 \pi-\theta) x}-e^{\theta x}\right)}{\left(\beta^{2}+x^{2}\right)^{2}\left(e^{2 \pi x}-1\right)} d x=\frac{1}{2 \beta^{2}}+\sum_{k=1}^{\infty} \frac{e^{i \theta k}}{(k+\beta)^{2}} .
\end{aligned}
$$

In particular, (20) and (21) imply that

$$
\begin{aligned}
& \int_{0}^{\infty} \frac{2 x e^{\pi x}}{\left(1+x^{2}\right)\left(e^{2 \pi x}-1\right)} d x=\frac{1}{2}+\sum_{n=1}^{\infty} \frac{(-1)^{n}}{n+1}=-\frac{1}{2}+\ln 2, \\
& \int_{0}^{\infty} \frac{4 x e^{\pi x}}{\left(1+x^{2}\right)^{2}\left(e^{2 \pi x}-1\right)} d x=\frac{1}{2}+\sum_{n=1}^{\infty} \frac{(-1)^{n}}{(n+1)^{2}}=\frac{\pi^{2}}{12}-\frac{1}{2} .
\end{aligned}
$$

Using (20) and (21) for $\beta=1$ and the particular cases, we find that

$$
M(\theta, T)=-1+\ln 2+\frac{1}{1+T^{2}} L_{2}(\theta, T)+\frac{T^{2}}{1+T^{2}}\left(\frac{\pi^{2}}{12}-\ln 2\right),
$$

where

$$
\begin{aligned}
& L_{2}(\theta, T)=\int_{0}^{\infty} \frac{x\left(e^{\theta x}+e^{(2 \pi-\theta) x}-2 e^{\pi x}\right)}{\left(1+x^{2}\right)\left(e^{2 \pi x}-1\right)} d x \\
& \quad+T \int_{0}^{\infty} \frac{2 x^{2}\left(e^{(2 \pi-\theta) x}-e^{\theta x}\right)}{\left(1+x^{2}\right)^{2}\left(e^{2 \pi x}-1\right)} d x+T^{2} \int_{0}^{\infty} \frac{2 x\left(e^{\theta x}+e^{(2 \pi-\theta) x}-2 e^{\pi x}\right)}{\left(1+x^{2}\right)^{2}\left(e^{2 \pi x}-1\right)} d x .
\end{aligned}
$$

We have to show that $L_{2}(\theta, T)>0$ for $\theta \in[0, \pi / 2]$ and $T \in \mathbb{R} . L_{2}(\theta, T)$ is a polynomial of the second degree with respect to $T$ with discriminant

$$
\begin{aligned}
\Delta_{2}(\theta)= & 4\left(\int_{0}^{\infty} \frac{x^{2}\left(e^{(2 \pi-\theta) x}-e^{\theta x}\right)}{\left(1+x^{2}\right)^{2}\left(e^{2 \pi x}-1\right)} d x\right)^{2} \\
& -8 \int_{0}^{\infty} \frac{x\left(e^{\theta x}+e^{(2 \pi-\theta) x}-2 e^{\pi x}\right)}{\left(1+x^{2}\right)^{2}\left(e^{2 \pi x}-1\right)} d x \int_{0}^{\infty} \frac{x\left(e^{\theta x}+e^{(2 \pi-\theta) x}-2 e^{\pi x}\right)}{\left(1+x^{2}\right)\left(e^{2 \pi x}-1\right)} d x .
\end{aligned}
$$

The Cauchy-Schwarz inequality implies

$$
\begin{aligned}
\int_{0}^{\infty} \frac{x\left(e^{\theta x}+e^{(2 \pi-\theta) x}-2 e^{\pi x}\right)}{\left(1+x^{2}\right)^{2}\left(e^{2 \pi x}-1\right)} d x \int_{0}^{\infty} \frac{x\left(e^{\theta x}+e^{(2 \pi-\theta) x}-2 e^{\pi x}\right)}{\left(1+x^{2}\right)\left(e^{2 \pi x}-1\right)} d x \\
\geq\left(\int_{0}^{\infty} \frac{x \sqrt{1+x^{2}}\left(e^{\theta x}+e^{(2 \pi-\theta) x}-2 e^{\pi x}\right)}{\left(1+x^{2}\right)^{2}\left(e^{2 \pi x}-1\right)} d x\right)^{2} .
\end{aligned}
$$


The inequality

$$
\begin{aligned}
\sqrt{2} \int_{0}^{\infty} \frac{x \sqrt{1+x^{2}}\left(e^{\theta x}+e^{(2 \pi-\theta) x}-2 e^{\pi x}\right)}{\left(1+x^{2}\right)^{2}\left(e^{2 \pi x}-1\right)} d x \\
\quad \geq \int_{0}^{\infty} \frac{x^{2}\left(e^{(2 \pi-\theta) x}-e^{\theta x}\right)}{\left(1+x^{2}\right)^{2}\left(e^{2 \pi x}-1\right)} d x, \quad \theta \in[0, \pi / 2],
\end{aligned}
$$

and (23) imply that $\Delta_{2}(\theta) \leq 0$ for $\theta \in[0, \pi / 2]$, which leads to $L_{2}(\theta, T) \geq 0$ for $\theta \in[0, \pi / 2]$ and $T \in \mathbb{R}$.

On the other hand, the inequality

$$
\sqrt{2\left(1+x^{2}\right)}\left(e^{\theta x}+e^{(2 \pi-\theta) x}-2 e^{\pi x}\right) \geq x\left(e^{(2 \pi-\theta) x}-e^{\theta x}\right)
$$

for $x \in(0, \infty)$ and $\theta \in[0, \pi / 2]$ implies (24). This inequality is equivalent to

$$
e^{(\pi-\theta) x} \geq \frac{\sqrt{2\left(1+x^{2}\right)}+x}{\sqrt{2\left(1+x^{2}\right)}-x}, \quad x \in[0, \infty), \theta \in[0, \pi / 2] .
$$

To check this last inequality, it is sufficient to consider the case $\theta=\pi / 2$, and consequently only

$$
e^{\frac{\pi}{2} x} \geq \frac{\sqrt{2\left(1+x^{2}\right)}+x}{\sqrt{2\left(1+x^{2}\right)}-x}, \quad x \in[0, \infty),
$$

must be proved. This is easily done using the derivative of the function

$$
h:[0, \infty) \rightarrow \mathbb{R}, \quad h(x)=e^{\frac{\pi}{2} x}-\frac{\sqrt{2\left(1+x^{2}\right)}+x}{\sqrt{2\left(1+x^{2}\right)}-x} .
$$

THEOREM 2. The largest value of $c$ for which the condition

$$
f \in \mathcal{A}, \quad \operatorname{Re}\left(f^{\prime}(z)+z f^{\prime \prime}(z)\right)>-c, \quad z \in U,
$$

implies the univalence of the function $f$, is $c=\frac{2 \ln 2-1}{2(1-\ln 2)}$.

Proof. According to the proof of Theorem 1, condition (25) implies that

$$
f(z)=z+2(1+c) \sum_{n=2}^{\infty} \frac{z^{n}}{n^{2}} \int_{0}^{2 \pi} e^{-i(n-1) t} d \mu(t)
$$

and

$$
\begin{aligned}
f^{\prime}(z) & =1+2(1+c) \sum_{n=1}^{\infty} \frac{z^{n}}{n+1} \int_{0}^{2 \pi} e^{-i n t} d \mu(t) \\
& =\left(1+2 \sum_{n=1}^{\infty} z^{n} \int_{0}^{2 \pi} e^{-i n t} d \mu(t)\right) *\left(1+(1+c) \sum_{n=1}^{\infty} \frac{z^{n}}{n+1}\right) .
\end{aligned}
$$


Because $1+2 \sum_{n=1}^{\infty} z^{n} \int_{0}^{2 \pi} e^{-i n t} d \mu(t) \in \mathcal{P}$, Lemma 2 implies that the necessary condition of univalence, $f^{\prime}(z) \neq 0$ for $z \in U$, holds if and only if

$$
\operatorname{Re}\left(1+(1+c) \sum_{n=1}^{\infty} \frac{z^{n}}{n+1}\right)>\frac{1}{2}, \quad z \in U .
$$

It is simple to prove that condition (26) is equivalent to

$$
\frac{1}{2(1+c)}+\sum_{n=1}^{\infty} \frac{(-1)^{n}}{n+1} \geq 0,
$$

which can be rewritten in the form

$$
c \leq \frac{2 \ln 2-1}{2(1-\ln 2)}
$$

Thus, by Theorem 1 the proof is finished.

4. Integral version of the result. Alexander's operator is defined by

$$
A(f)(z)=\int_{0}^{z} \frac{f(t)}{t} d t, \quad z \in U
$$

Let $R_{c}=\left\{f \in \mathcal{A}: \operatorname{Re}\left(f^{\prime}(z)\right)>-c, z \in U\right\}$. Using this notation, Theorem 1 becomes:

THEOREM 3. If $c=\frac{2 \ln 2-1}{2(1-\ln 2)}$ then $A\left(R_{c}\right) \subseteq S^{*}$, and the result is sharp.

\section{References}

[1] R. M. Ali, A subclass of starlike functions, Rocky Mountain J. Math. 24 (1994), 447-451.

[2] P. T. Mocanu, On starlike images by Alexander integral, Babeş-Bolyai Univ., Fac. Math. Phys., Seminar on Functional Equations, Approximation and Convexity, Preprint 6 (1987), 245-250.

[3] S. Ponnusamy, On starlikeness of certain integral transforms, Ann. Polon. Math. 56 (1992), 227-232.

[4] St. Ruscheweyh, Convolution in Geometric Function Theory, Les Presses Univ. de Montréal, Montréal, 1982.

[5] R. Singh and S. Singh, Starlikeness and convexity of certain integrals, Ann. Univ. Mariae Curie-Skłodowska Sect. A 35 (1981), 145-148.

Sapientia - Hungarian University of Transilvania

str. Sighişoarei, nr. 1c

540053 Corunca, Judeţul Mureş

Romania

E-mail: rszasz@ms.sapientia.ro 